\title{
Structural and functional studies of the $\beta$-barrel assembly machinery
}

\author{
Jeremy Bakelar and Nicholas Noinaj \\ Dept. of Biological Sciences, Purdue University, West Lafayette, IN
}

$\beta$-barrel outer membrane proteins (OMPs) are found within the outer membranes (OM) of Gramnegative bacteria and serve critical functions including nutrient import, cell signaling, and protection. In the final stage of their biogenesis, nascent OMPs are folded and inserted into the OM by a conserved multi-protein complex called the $\beta$-barrel assembly machinery (BAM) complex. Although essential, the mechanistic details of BAM complex function have remained elusive. Recently, we and others reported structures of fully assembled BAM in differing conformational states. These studies have provided the molecular blueprint detailing proteinprotein interactions within the complex and have revealed new details about BAM, suggesting a dynamic mechanism that may use conformational changes to assist in the biogenesis of new OMPs. Building on this recent progress we have performed a robust structure guided mutagenesis and functional investigation of the central component BamA, which has identified protein binding interfaces and conformational changes important for BAM function, including the large conformational switch of the BamA $\beta$-barrel domain. 